\title{
How should be the Surgical Treatment Approach during the COVID-19 Pandemic in Patients with Gastrointestinal Cancer?
}

\author{
(D) Mustafa Fevzi Celayir, (1) Nurcihan Aygun, (1) Mert Tanal, (1) Hakan Mustafa Koksal, (i) Evren Besler, \\ (D) Mehmet Uludag
}

Department of General Surgery, Sisli Hamidiye Etfal Training and Research Hospital, Istanbul, Turkey

\begin{abstract}
COVID-19 was first seen in China at the end of December 2019. COVID-19 is a novel type of coronavirus that is defined as SARS$\mathrm{CoV}-2$, which can be mild or severe in the lungs, causing acute respiratory infection. The disease was first presented in the literature as Coronavirus Disease 2019 (COVID-19) in February 2020. The disease spread rapidly and was declared as a pandemic by the World Health Organization (WHO) on March 11, 2020. There have been approximately 7734185 reported cases, and 412369 reported deaths to date (09/June/2020).

As COVID-19 spread in the world and our country, hospitals struggling with this disease have also become risky areas for transmitting the disease. Health workers also have a high risk of viral contamination from direct contact of droplets and surfaces. Personal protective equipment (PPE), such as masks, coveralls, gloves, face shields and/or goggles, are mandatory. The aim is to spread the flow of cases requiring hospitalization over time, thereby preventing possible accumulation in hospitals.

All non-urgent procedures, such as elective surgeries and diagnostic interventions, were significantly affected. The hospitalization procedures were mostly allocated to patients with COVID-19 infection, and surgical operations were postponed. Only urgent surgical cases and oncological surgeries that cannot be postponed were performed during this pandemic process.

Patients followed by oncology are immunosuppressed both because of the disease itself and the side effects of chemotherapy and/or radiotherapy taken. This makes patients more susceptible to infections, and the prognosis of infections in these patients is worse and more destructive. Cancer patients are almost twice as likely to catch COVID-19 compared to the general population. The choice of surgical procedures and perioperative management of the patients with malignancy has become even more important in the COVID-19 pandemic. In this study, we analyzed the treatment processes of our patients with malignancy that underwent a surgical oncological procedure during this pandemic.

Keywords: Cancer; COVID-19; general surgery; oncological emergencies; SARS CoV-2.
\end{abstract}

Please cite this article as "Celayir MF, Aygün N, Tanal M, Köksal HM, Besler E, Uludağ M. How should be the Surgical Treatment Approach during the COVID-19 Pandemic in Patients with Gastrointestinal Cancer? Med Bull Sisli Etfal Hosp 2020;54(2):136-141".

Ciston oronavirus family (CoV) is a quite large family of viruses. It is a common cause of infections in humans and animals. In the microscopic examination, the virus resembles a crown. That is why it is called Corona, which means "crown" or "ring of light" in Latin. It can easily be transmitted from animals to humans. Although bats or pangolin is considered to be the first source, the source is not yet known. ${ }^{[1]}$

Coronavirus-19 (COVID-19), first reported in Wuhan, Hubei Province, the People's Republic of China on December 31, 2019 , is a highly contagious RNA virus with devastating ef-

Address for correspondence: Mustafa Fevzi Celayir, MD. Sisli Hamidiye Etfal Egitim ve Arastirma Hastanesi, Genel Cerrahi Klinigi, Istanbul, Turkey Phone: +90 5322418245 E-mail: fcelayir@gmail.com

Submitted Date: June 09, 2020 Accepted Date: Jun 16, 2020 Available Online Date: June 16, 2020

${ }^{\circ}$ Copyright 2020 by The Medical Bulletin of Sisli Etfal Hospital - Available online at www.sislietfaltip.org

OPEN ACCESS This is an open access article under the CC BY-NC license (http://creativecommons.org/licenses/by-nc/4.0/). 
fects for worldwide health systems by causing severe acute respiratory syndrome-Coronavirus-2 (SARS-CoV-2). ${ }^{[2]}$ Its definitive treatment and vaccine have not yet been found, although ongoing worldwide studies are present.

Coronavirus-infected people may experience a wide range of illnesses from flu to severe outbreaks of the Middle East Respiratory Syndrome (MERS-CoV) and Severe Acute Respiratory Syndrome (SARS-CoV) and may have complaints of the respiratory system, gastrointestinal system, liver, kidney damage, and even dermatological, neurological or psychological problems in various degrees.

Initial reports suggest that COVID-19 is associated with severe disease that requires intensive care in approximately $5 \%$ of the proven infections. ${ }^{[3]}$ Individuals with COVID-19 usually develop signs and symptoms, such as mild respiratory illness and persistent fever, an average of 5-6 days after infection (range, 1-14 days). The most common clinical symptoms of COVID-19 are fever, cough, shortness of breath, fatigue, and rarely, diarrhea and vomiting. ${ }^{[4]}$

COVID-19 is transmitted by direct human to human exposure (respiratory droplets) or direct contact with contaminated surfaces (viral particles). Thus, frequent hand hygiene and social distance (more than 1.5-2 m) should be maintained when dealing with COVID-19 positive patients, and if close contact happens with the patient, PPE should be worn, because the healthcare workers should not get infected and should not infect other patients and healthcare workers by being a carrier.

COVID-19 may lead to symptoms ranging from clinically asymptomatic infection to severe respiratory failure. Laboratory findings include leukopenia and lymphopenia. Lymphopenia is its most important feature. Both lactate dehydrogenase (LDH) and creatinine kinase (KK) may increase. Also, liver enzymes, such as alanine aminotransferase (ALT) or aspartate aminotransferase (AST), can be abnormal. ${ }^{\text {[5] }}$ Real-time PCR tests (Real time-polymerase chain reaction RT-PCR) have highly sensitive diagnostic accuracy. Irregular ground-glass opacities and irregular consolidation scattered in the middle and outer region of the lungs seen on computed tomography of the thorax are diagnostic in most patients. ${ }^{[6]}$

The average time between the onset of symptoms and hospitalization in the intensive care unit (ICU) is 9 to 10 days, which indicates a gradual deterioration in most cases. Acute respiratory distress syndrome (ARDS) and respiratory support requirement developing in two-thirds of patients were the most important ICU indications. ${ }^{[7]}$

Cancer patients are vulnerable. They are much more susceptible to infections due to immunosuppression by both medical and surgical treatment. ${ }^{[8]}$ Therefore, these patients are at a higher risk for COVID-19. They were nearly three times more likely to have severe or critical illness (34\%), be admitted to an ICU (19\%) or be put on a ventilator (10\%). Moreover, people with cancer are about twice as likely to die as COVID-19 patients without cancer (11\% versus 5\%, respectively). Patients with progressive cancer died at a numerically higher rate without ICU admission than among patients who were admitted to an ICU, and the reverse pattern was seen for patients in remission. ${ }^{[9]}$ In this study, we investigated how we can manage cancer patients that we could not postpone their operations in the COVID-19 pandemic.

\section{Management and Ethical Issues of Oncological Surgery under Pandemic Conditions}

While planning the surgery of oncological patients in a pandemic, it is necessary to decide the urgency and stage of cancer and to implement surgical interventions by taking necessary precautions. Due to the COVID-19 outbreak, it may be necessary to change the order of oncological procedures. For example, in a patient in whom an elective surgery decision due to breast cancer would be made before the pandemic, medical treatment may be brought forward to postpone the surgery. ${ }^{[10]}$

Nowadays, the number of patients with COVID-19 is expected to increase more in certain periods, maybe the number of cases will decrease, and then make a peak again until the pandemic ends.

When the need for surgical treatment occurs in cancer patients, treatments should be limited to life-threatening malignancies or processes that require urgent/emergent surgery. Elective surgical procedures should be postponed until favorable conditions are provided because there are three major concerns of why malignancies can increase the risk of getting the virus:

1. Many patients with the diagnosis of cancer have received or are currently receiving treatments suppressing the immune system.

2. Cancer may negatively affect the functions of the lung or kidney. Reduced organ functions make the immune system vulnerable to extremely high-risk diseases, such as COVID-19.

3. Hematological cancers negatively affect the functioning of the immune system. These patients may be particularly sensitive to COVID-19. Especially, patients who have bone marrow transplantation appear to be at high-risk group.

A recent article published in China suggests that cancer patients are more likely to become infected with COVID-19 due to suppressed immune systems that arise from their 
chemotherapy regimens and surgery. The article also emphasizes that these COVID-19 infected patients are more likely to develop severe infiltrations (39\% versus $8 \%$ ). According to articles published before the pandemic, the risk of developing a severe infection was higher in patients who received chemotherapy or undergone surgery than patients who did not receive either $75 \%$ vs. $43 \%$, respectively. ${ }^{[8,9]}$ For surgical oncology, this period differentiates into three parts as follows: preoperatively, during the surgery, postoperatively.

In elective cases, going to the hospital during the COVID-19 outbreak may put the patient at risk of infection. Therefore, it is important to reduce the time the patient with malignancy spending in the hospital. ${ }^{[11]}$

To minimize face-to-face communication and direct contact, telephone or video-conference calls will be appropriate, especially for pre-treatment meetings and follow-up appointments. Necessary blood tests can be performed in clean hospitals with a low patient burden. Drugs can either be sent home or a pick-up point may be arranged. Electronic prescriptions for patients taking oral oncological treatments should have an extended period to prevent patients from getting to hospitals just for the prescription. When neoadjuvant radiotherapy is needed, short-term radiotherapies rather than long-term should be preferred to get less contact with hospital setting. ${ }^{[12]}$

As the outbreak is unlikely to resolve within weeks, various guidelines and classifications have emerged in the clinical practice of surgical oncologists. ${ }^{[13]}$ The main problem here is to deal with the problems that arise from the delays in cancer patients and to protect these patients from COVID-19. ${ }^{[14]}$ ESMO (European Society of Medical Oncology) prefers a gradual approach to the management of cancer patients during the COVID-19 outbreak. They recommend differentiating patients by dividing them into the following groups by considering the patient's current condition, the urgency of the surgical oncology procedure, the potential risk of COVID-19, and the available resources for safely treating the patient with malignancy. ${ }^{[15]}$

1. High priority: This group includes patients who have a life-threatening or clinically imbalanced condition and/ or planned treatment would result in a significant benefit (for example, prolonging life or improving quality of life) where treatment becomes a priority. ${ }^{[15]}$

2. Medium priority: This group includes patients who are not in life-threatening, but a severe condition, where a short delay in treatment may be considered. However, a delay of more than 6-8 weeks may potentially affect the benefit and/or outcome obtained, which makes the treatment a medium priority. ${ }^{[15]}$
3. Low priority: This group includes patients who are stable in whom any treatment can be safely delayed during the COVID-19 pandemic. It also includes patients who are unlikely to have a significant benefit from the planned intervention (unlikely to prolong life or to improve the quality of life), which all makes the treatment a low priority. ${ }^{[15]}$

Each patient should be examined by a multidisciplinary team to decide whether any changes in treatment are required. The patient should be informed of whether to change the management of the current disease, how and why. While doing all this, the deontological principles should be kept in mind.

Organ-specific cancer approaches SSO (Society of Surgical Oncology):

1-Upper Gastrointestinal:

For gastric and esophageal cancer: ${ }^{[16]}$

cT1a lesions - Endoscopic resection if suitable

cT1b cancers - Resection

cT2 or above and node-positive tumors-total neoadjuvant therapy

Consider proceeding directly to neoadjuvant therapy without staging laparoscopy due to concerns about laparoscopic surgery in patients with COVID-19 and increased use of resources; if staging laparoscopy performed, consider minimizing the use of personal protective equipment and staff involved and/or exposed in procedure using appropriate pneumoperitoneum risk-reduction strategies. ${ }^{[16]}$

Patients completing neoadjuvant chemotherapy may remain on chemotherapy if responding to and tolerating therapy and resources do not support proceeding to resection; if the patient is not responding to systemic therapy, consider resection and/or referral. Consider treating patients with gastric outlet obstruction or hemorrhage with endoscopic measures to allow for enteral nutrition or control of bleeding; proceed to surgery if measures fail. Percutaneous endoscopic gastrostomy (PEG) may be recommended to eligible patients with nutritional problems Consider short-term deferral of surgery in less biologically aggressive cancers, such as gastrointestinal stromal tumors, unless symptomatic or bleeding. ${ }^{[16]}$

For hepatopancreatobiliary cancer: ${ }^{[16]}$

Potentially curable pancreatic adenocarcinoma (included those who have completed neoadjuvant therapy and those with biliary/gastric obstruction), pancreatic cystic lesions with confirmed high grade dysplasia, duodenal cancer, ampullary cancer, potentially curable large/multifocal hepatocellular carcinoma and cholangiocarcinoma should undergo surgical resection. When resources are limited; the 
patient can prolong neoadjuvant therapy when responding and tolerating. If pandemic escalates, only emergent life-saving interventions should be performed (e.g., drainage for cholangitis, gastric outlet obstruction and bleeding management). ${ }^{[16]}$

\section{2- Lower Gastrointestinal:: ${ }^{[16]}$}

Colorectal cancer ${ }^{[16]}$

All cancers in polyps or another early-stage disease - defer surgery

Obstructed (divert only if rectal), perforated, or acutely transfusion-dependent patients - proceed with surgery

Non-metastatic colon cancer - proceed with definitive surgery ${ }^{[16]}$

Consider all options for neoadjuvant therapy, including utilization of total neoadjuvant therapy for rectal cancer and consider neoadjuvant chemotherapy for locally advanced and metastatic colon cancer. For rectal cancer neoadjuvant therapy, highly consider short course regimen ( 5 days of 5 Gy) compared to long course chemoradiation therapy. ${ }^{[16]}$

For locally advanced rectal cancer, delay surgery for 12-16 weeks following neoadjuvant therapy. In patients with a complete response after neoadjuvant therapy, the "wait and see approach" cam also be suggested by closely monitoring the patient and discussing the situation with the patient. ${ }^{[17]}$

Concerning surgery, long-term neoadjuvant therapy and waiting time can provide an advantage to the patient and the doctor during the pandemic period.

\section{Organizations Rearranged in the Pandemic for Surgery: Before the Operation}

The patient's file should be examined in a safe, clean area before communicating with the patient. Then, the patient should be examined with other intervening doctors, the information should be taken from the family about other concomitant diseases not reflected in the patient's medical history, and all these data should be recorded. ${ }^{[18]}$

If the patient is electively prepared, it will be an important advantage for the patient to isolate himself/herself for 14 days.

Since PCR swab tests yield late results in a patient undergoing surgery, thorax CT should be a priority. By this, lung lesions specific to COVID-19 infection (i.e., ground-glass opacification) may show the presence of viral infection to surgeons more rapidly than PCR tests. Thorax CT is $97 \%$ and $75 \%$ sensitive with positive and negative PCR tests, respectively. ${ }^{[19]}$

In oncological emergent surgeries, the preoperative period is provided within hospital standards. The approach in these patients is similar to approaches to surgeries of patients with suspected or diagnosed COVID-19 infection. ${ }^{[20]}$

\section{During the Operation}

There must be minimum staff in the operating room. The OR door must be kept closed at all times. If the patient is COVID-19 diagnosed or highly suspected, complete PPE should be worn. These should be surgical caps, coveralls, N95 masks undercovered with surgical masks, double-layer gloves, face shields or goggles, and both dressing and removal should be performed in accordance with the guidelines. ${ }^{[12]}$

If the patient has a negative thorax $\mathrm{CT}$ and/or negative PCR swab test, surgical boxes may be worn instead of coveralls. As a general surgeon and the author of this article who had COVID-19 infection and survived, I emphasize that we recommend following these rules of protection, as there is no definite proposal on this subject.

\section{Open or Laparoscopic Surgery?}

If an abdominal surgery is going to be performed in the surgery, the preference of our hospital is open surgery because the risk of transmission of COVID-19 is high in laparoscopic surgery due to the viral pathogens in the peritoneal cavity. However, with a recent article, this issue has become controversial. This article showed an interesting result regarding the presence of COVID-19 pathogens in the peritoneal fluid. This case revealed that COVID-19 was not detected in peritoneal fluid and peritoneal washings in a patient infected with COVID-19. This study provides novel preliminary data in the investigation of COVID-19 transmission from laparoscopy-related aerosolization. ${ }^{[21]}$

The surgery is performed using the standard surgical techniques specific to the disease. Blood transfusions can be performed if needed because there is no evidence of COVID-19 transmission by transfusion. The aerosol and particles released during and after the surgery (leaks or inhalation) may contaminate healthcare workers and all surfaces in the operating room. However, if fixed pressure insufflators, closed-circuit smoke absorption system (host system would be more appropriate), a negative pressure operating room and carbon dioxide filter are used to discharge the smoke to reduce the aerosol effects of insufflation, laparoscopic surgery will be a preference in the COVID-19 pandemic with its known advantages of shorter length of stay, early recovery, and discharge. ${ }^{[22]}$ Society of American Gastrointestinal and Endoscopic Surgeons (SAGES) has recommended the use of an active smoke evacuator connected to a proper filter by for laparoscopic or robotic procedures during the COVID-19 pandemic. ${ }^{[23]}$

Chen et al. stated that surgical operation should be minimized to prevent cross-infection, and surgical interventions should be postponed for benign tumors. They suggested that multidisciplinary and non-surgical anti-tumor therapy 
should be prioritized. Neoadjuvant chemotherapy can also be a good choice for advanced gastrointestinal tumors. The nutritional status of the patient can be provided with solutions, such as a nasogastric catheter, PEG, and stenting. All invasive interventions should be made in a designated isolation room. A protective enterostomy is also a good option in lower digestive system surgeries. ${ }^{[24]}$ If the interventional nutrition procedures are applied in COVID-19 positive patients, total parenteral nutrition can be given to the patient, at least in the recovery period of COVID-19.

\section{After the Operation}

Disinfection procedures for the operating rooms postoperatively are described in detail in COVID-19 Prevention and Treatment Guideline written by Cai Hongliu et al. ${ }^{[25]}$ The surgical instruments used during procedures of $\mathrm{CO}$ VID-19 positive or suspected patients are recommended to be sterilized separately. In addition to standard procedures, solutions containing $0.1 \%$ sodium hypochlorite should be added to the sterilization. Operating room (OR) covers and shirts should be put directly into special bags or boxes separated, or preferably, disposable laundries should be used, if possible. Standard $70^{\circ} \mathrm{C}$ laundry processes are sufficient to eliminate the virus. The specimens are submitted to the Department of Pathology with the same former procedures. The disinfection time between cases in OR should be longer than 30 minutes. The operating room should be kept closed for at least two hours, and the next operation should begin after the laminar flow and ventilators are opened. Meanwhile, hydrogen peroxide nebulization and ultraviolet light sterilization are also recommended. ${ }^{[26]}$

\section{Patient Care and Follow-up Postoperatively}

Patients not requiring intensive care postoperatively are taken to their patient rooms after they recovered. If the patient does not have COVID-19 suspicion or diagnosis, surgical follow-up is performed in surgery clinics clean for COVID-19, with the rules before the pandemic. However, if the patient has a diagnosis confirming COVID-19 infection, antiviral therapies may be applied. ${ }^{[27]}$ There is still no consensus on the antiviral therapies for COVID-19 infection, and the management is updated daily. Since the side effects and effectiveness of the treatments are uncertain, at least informed consent must be obtained verbally from the patient before the antiviral treatment.

\section{Telemedicine}

Telemedicine or tele-oncology is a new tool in the long-term follow-up of cancer patients during the pandemic so that patients can be followed up remotely effectively and cheaply without the risk of infection. In addition, Coronavirus Tele
Rehabilitation Support Program (KOREH) has been launched in Turkey and provides remote physical therapy services to the patients at their homes. This approach was quickly accepted all over the world. The only problem is that the sense of clinical feeling cannot be established with the patients. ${ }^{[28]}$

\section{Conclusion}

Since the first reported COVID-19 cases, the health systems faced with a great load. Preventing infection and reducing the speed of infection rate are the main goals. Thus, many elective surgical procedures have been canceled or postponed due to COVID-19. Instead, only emergent surgical interventions and oncological emergent surgeries have been performed.

To maintain the sustainability of the health system, the protection of healthcare providers is also a priority. On the other hand, appropriate treatment should be provided to patients who require urgent health care. Patients with malignancies are included in this group.

Just after the first cases reported in Turkey, our hospitals reorganized their staff to use the workforce effectively. Our national scientific committee established by the Ministry of Health, Turkey, worked as an example for us by closely monitoring and evaluating information from the world and our country in the pandemic.

It has been shown that oncological emergency patients can always be operated safely if necessary precautions are taken, and current recommendations in the literature are applied in this difficult period. Gastrointestinal cancers, which can be postponed, should be placed on the waiting list until the pandemic ends. However, in this process, patients should not be left to their fate; they should be followed by telemedicine with a multidisciplinary approach. Our hope is to overcome this pandemic with the least mortality and to return gradually and selectively to the former normal life as soon as possible.

\section{Disclosures}

Peer-review: Externally peer-reviewed.

Conflict of Interest: None declared.

Authorship Contributions: Concept - M.F.C., M.U.; Design - M.F.C.; Supervision - M.U.; Materials - N.A.; Data collection \&/or processing - M.T., E.B.; Analysis and/or interpretation - H.M.K., M.T.; Literature search - M.F.C.; Writing - M.F.C.; Critical review - M.U.

\section{References}

1. Killerby ME, Biggs HM, Haynes A, Dahl RM, Mustaquim D, Gerber $\mathrm{SI}$, et al. Human coronavirus circulation in the United States 2014 2017. J Clin Virol 2018;101:52-6. [CrossRef]

2. Gosain R, Abdou Y, Singh A, Rana N, Puzanov I, Ernstoff MS. CO- 
VID-19 and Cancer: a Comprehensive Review. Curr Oncol Rep 2020;22:53. [CrossRef]

3. Murthy $S$, Gomersall CD, Fowler RA. Care for Critically III Patients With COVID-19. JAMA 2020 Mar 11 [Epub ahead of print], doi: 10.1001/jama.2020.3633. [CrossRef]

4. Lima CMAO. Information about the new coronavirus disease (COVID-19). Radiol Bras 2020;53:V-VI. [CrossRef]

5. Rodriguez-Morales AJ, Cardona-Ospina JA, Gutiérrez-Ocampo $E$, Villamizar-Peña R, Holguin-Rivera Y, Escalera-Antezana JP, et al; Latin American Network of Coronavirus Disease 2019-COVID-19 Research (LANCOVID-19). Clinical, laboratory and imaging features of COVID-19: A systematic review and meta-analysis. Travel Med Infect Dis 2020;34:101623. [CrossRef]

6. Hu Q, Guan H, Sun Z, Huang L, Chen C, Ai T, et al. Early CT features and temporal lung changes in COVID-19 pneumonia in Wuhan, China. Eur J Radiol 2020 Apr 19 [Epub ahead of print], doi: 10.1016/j.ejrad.2020.109017. [CrossRef]

7. Yeoh CB, Lee KJ, Rieth EF, Mapes R, Tchoudovskaia AV, Fischer GW, et al. COVID-19 in the Cancer Patient. Anesth Analg. 2020 Apr 22 [Epub ahead of print], doi: 10.1213/ANE.0000000000004884.

8. Liang W, Guan W, Chen R, Wang W, Li J, Xu K, et al. Cancer patients in SARS-CoV-2 infection: a nationwide analysis in China. Lancet Oncol 2020;21:335-7. [CrossRef]

9. Highleyman L. Cancer Patients With COVID-19 May Have Higher Risk of Severe IIIness and Death Cancer Health. Available at: https://www.cancerhealth.com/article/cancer-patients-covid19may-higher-risk-severe-illness-death. Accessed Jun 10, 2020.

10. Citgez B, Yigit B, Capkınoglu E, Yetkin SG. Management of breast cancer during the COVID-19 pandemic. Sisli Etfal Med Bul 2020 Jun, [Epub ahead of print], doi: 10.14744/SEMB.2020.23326.

11. Liu Z, Zhang Y, Wang X, Zhang D, Diao D, Chandramohan K, et al. Recommendations for Surgery During the Novel Coronavirus (COVID-19) Epidemic. Indian J Surg 2020 Apr 11 [Epub ahead of print], doi: 10.1007/s12262-020-02173-3. [CrossRef]

12. Aygun N, Iscan Y, Ozdemir M, Soylu S, Aydın OU, Sormaz iC et al. Endocrine surgery during the COVID-19 pandemic: Recommendations from Endocrine Surgery Society of Turkey. Sisli Etfal Med Bul 2020 Jun [Epub ahead of print], doi: 10.14744/ SEMB.2020.65902. [CrossRef]

13. Ghignone F, Mohan HM, Montroni I. Cancer surgery in a time of COVID-19: Many questions, few certainties. Eur J Surg Oncol 2020;46:1196-7. [CrossRef]

14. Grass F, Behm KT, Duchalais E, Crippa J, Spears GM, Harmsen WS, et al. Impact of delay to surgery on survival in stage I-III colon cancer. Eur J Surg Oncol 2020;46:455-61. [CrossRef]

15. ESMO. Cancer care during the Covid-19 pandemic: An ESMO guide for patients. Available at: https://www.esmo.org/for-patients/patient-guides/cancer-care-during-the-covid-19-pan- demic. Accessed Jun 10, 2020.

16. Society of Surgical Oncology. Resource for Management Options of Colorectal Cancer During COVID-19. Available at: https://www. surgonc.org/wp-content/uploads/2020/03/Colorectal-Resourceduring-COVID-19-3.30.20.pdf. Accessed Jun 10, 2020.

17. Akyol C, Koç MA, Utkan G, Yıldız F, Kuzu A. The COVID 19 Pandemic and Colorectal Cancer: 5W1H - What Should We Do to Whom, When, Why, Where and How? Turk J Colorectal Dis 2020;30:67-75.

18. Kamer E, Çolak T. What to do when a patient infected with COVID-19 needs an operation: A pre-surgery, peri-surgery and postsurgery guide. Turk J Colorectal Dis 2020;30:1-8. [CrossRef]

19. Tomulescu V, Surlin V, Scripcariu V, Bintintan V, Duta C, Calu V, et al. Colorectal Surgery in Romania during the COVID-19 Pandemic. Chirurgia 2020;115:129. [CrossRef]

20. Kim H, Hong H, Yoon SH. Diagnostic Performance of CT and Reverse Transcriptase-Polymerase Chain Reaction for Coronavirus Disease 2019: A Meta-Analysis. Radiology 2020 Apr 17 [Epub ahead of print], doi: 10.1148/radiol.2020201343. [CrossRef]

21. Ngaserin SHN, Koh FH, Ong BC, Chew MH. COVID-19 not detected in peritoneal fluid: A case of laparoscopic appendicectomy for acute appendicitis in a COVID-19-infected patient. Langenbecks Arch Surg 2020;405;353-5. [CrossRef]

22. Karaca AS, Özmen MM, Uçar AD, Yastı AÇ, Demirer S. COViD-19'lu Hastalarda Genel Cerrahi Ameliyathane Uygulamaları. Turk J Surg 2020;36:VI-X. [CrossRef]

23. Wexner SD, Cortés-Guiral D, Gilshtein H, Kent I, Reymond MA. COVID-19: impact on colorectal surgery. Colorectal Dis. 2020 May [Epub ahead of print], doi: 10.1111/codi.15112. [CrossRef]

24. Chen N, Zhou M, Dong X, Qu J, Gong F, Han Y, et al. Epidemiological and clinical characteristics of 99 cases of 2019 novel coronavirus pneumonia in Wuhan, China: a descriptive study. Lancet 2020;395:507-13. [CrossRef]

25. Handbook of COVID-19 Prevention and Treatment. Available at: https://esge.org/documents/Handbook_of_COVID-19_Prevention_and_Treatment.pdf. Accessed Jun 10, 2020.

26. Tao KX, Zhang BX, Zhang P, Zhu P, Wang GB, Chen XP; General Surgery Branch of Hubei Medical Association; General Surgery Branch of Wuhan Medical Association. [Recommendations for general surgery clinical practice in novel coronavirus pneumonia situation]. Zhonghua Wai Ke Za Zhi. 2020 Feb 14 [Epub ahead of print], doi: 10.3760/cma.j.issn.0529-5815.2020.0001.

27. Wax RS, Christian MD. Practical recommendations for critical care and anesthesiology teams caring for novel coronavirus (2019nCoV) patients. Can J Anaesth 2020;67:568-76. [CrossRef]

28. Shirke MM, Shaikh SA, Harky A. Tele-oncology in the COVID-19 Era: The Way Forward? Trends Cancer 2020 May 27 [Epub ahead of print], doi: 10.1016/j.trecan.2020.05.013. [CrossRef] 